\title{
Upper Quantum Lyapunov Exponent and Anosov relations for quantum systems driven by a classical flow
}

\author{
O. Sapin, H. R. Jauslin \\ Laboratoire de Physique CNRS - UMR 5027 \\ Université de Bourgogne \\ BP 47870, F-21078 Dijon, France \\ Stefan Weigert \\ Department of Mathematics \\ University of York \\ Heslington YO10 5DD, UK
}

\begin{abstract}
We generalize the definition of quantum Anosov properties and the related Lyapunov exponents to the case of quantum systems driven by a classical flow, i.e. skew-product systems. We show that the skew Anosov properties can be interpreted as regular Anosov properties in an enlarged Hilbert space, in the framework of a generalized Floquet theory. This extension allows us to describe the hyperbolicity properties of almost-periodic quantum parametric oscillators and we show that their upper Lyapunov exponents are positive and equal to the Lyapunov exponent of the corresponding classical parametric oscillators. As second example, we show that the configurational quantum cat system satisfies quantum Anosov properties.
\end{abstract}




\section{Introduction}

Anosov properties and Lyapunov exponents are well-established characterization of classical dynamics and it is natural to search for similar concepts applicable to quantum dynamics. Several definitions have been given in the literature (see 14, 17, 18, 1, 24, 21, 13, 15, 20, 16, 6, and the references therein).

In Ref. 14, Majewski and Kuna defined a quantum Lyapunov exponent for $N$-level quantum systems. Later ${ }^{1}$, Emch, Narnhofer, Sewell and Thirring [1, 24, 20] proposed an axiomatic framework which allows one to define an Anosov property for quantum mechanical systems. However, the resulting definition of a quantum Lyapunov exponent is limited since it only applies to systems with a globally constant hyperbolicity property.

In Ref. [6], the upper Lyapunov exponent for quantum systems in the Heisenberg representation has been defined, close in spirit to definitions given in Refs. 14, 1. Its usefulness has been illustrated with the example of the parametric quantum oscillator with periodic time dependence. Moreover, it was shown that whenever its upper Lyapunov exponent is positive, the system satisfies the discrete quantum Anosov relations defined by Emch, Narnhofer, Sewell and Thirring [1, 24, 20].

In this paper we extend the study to systems described by a Hamiltonian operator of the form $H\left(\varphi^{t}(\theta)\right.$ ) (with $\varphi^{t}$ a flow on a space $\mathcal{M}$ ), which will be referred to as quantum skew-product system. We generalize the definition of Anosov relations so that it applies to this type of system. As in the case of Floquet theory 4, 3, 28, 5, 2, it is possible to make quantum skewproduct systems autonomous by embedding the dynamics in a larger Hilbert space. The Anosov relations for quantum skew-product systems correspond to the Anosov relations of the associated system in this enlarged Hilbert space. We consider the parametric oscillator as an example. We show that the quantum parametric oscillator verifies the Anosov relations for quantum skew-product systems if its upper Lyapunov exponent is positive and the corresponding classical dynamics is reducible. Thus the quantum parametric oscillator discussed in [6] is an Anosov quantum skew-product system. As a second example we consider the configurational quantum cat system [25, 26, 27, with periodic boundary conditions, which amounts to a system with compact configuration space.

This paper is organized in the following way: In Section 2 we recall the definition of the upper Lyapunov exponent and of the Anosov properties for a quantum system describing the motion of a particle. In Section 3, we present the formalism of quantum skew-product systems and the enlarged Hilbert space which allows one to turn the system into an autonomous one. We

\footnotetext{
${ }^{1}$ Erratum to Ref. [6]: The chronology of the Refs. [14 and [1] as described in Refs. [6] by two of the present authors is erroneous. To our knowledge the works of [14 and [1] were developped independently, while [14] was published before [1].
} 
propose a definition of the quantum Anosov properties for quantum skewproduct systems in Section 4. Finally, we treat the example of the almostperiodic quantum parametric oscillator and the configurational quantum cat system in Section 5.

\section{Upper Lyapunov exponents and quantum Anosov relations}

A quantum mechanical particle in one dimension is described by coordinate and momentum operators $\hat{x}$ and $\hat{p}$ which satisfy the Heisenberg commutation relation (we choose the units such that $\hbar=1$ ):

$$
[\hat{x}, \hat{p}]=i .
$$

It is convenient to consider the $C^{*}$-algebra generated by Weyl operators:

$$
W(\beta, \gamma)=\exp [i(\beta \hat{x}+\gamma \hat{p})], \quad \forall \beta, \gamma \in \mathbb{R} .
$$

These operators satisfy the Weyl form of the commutation relations:

$$
\begin{aligned}
W(\beta, \gamma)^{\dagger} & =W(-\beta,-\gamma) \\
W(\beta, \gamma) W\left(\beta^{\prime}, \gamma^{\prime}\right) & =e^{-\frac{i}{2}\left(\beta \gamma^{\prime}-\gamma \beta^{\prime}\right)} W\left(\beta+\beta^{\prime}, \gamma+\gamma^{\prime}\right) .
\end{aligned}
$$

More abstractly, if the phase space is a real symplectic space $V$ with symplectic form $\sigma$, the $C^{*}$-algebra $\mathcal{W}$ of canonical commutation relations over $(V, \sigma)$ is defined as the $C^{*}$-algebra generated by elements $\{W(\underline{\alpha}) \mid \underline{\alpha} \in V\}$ such that

$$
\begin{aligned}
W(\underline{\alpha})^{\dagger} & =W(-\underline{\alpha}), \\
W(\underline{\alpha}) W\left(\underline{\alpha^{\prime}}\right) & =e^{-\frac{i}{2} \sigma\left(\underline{\alpha}, \underline{\alpha}^{\prime}\right)} W\left(\underline{\alpha}+\underline{\alpha}^{\prime}\right) .
\end{aligned}
$$

In this paper we consider only phase spaces $V$ of finite dimension $2 n$, with the usual symplectic form

$$
\sigma\left(\underline{\alpha}, \underline{\alpha}^{\prime}\right)=\alpha_{x}^{\top} \alpha_{p}^{\prime}-\alpha_{p}^{\top} \alpha_{x}^{\prime} \quad \forall \underline{\alpha}=\left(\begin{array}{c}
\alpha_{x} \\
\alpha_{p}
\end{array}\right), \underline{\alpha}^{\prime} \in \mathbb{R}^{2 n},
$$

where $\alpha_{x}^{\top}$ denotes the transposed of $\alpha_{x}$. Hence, the Weyl operators can be written as:

$$
W(\underline{\alpha})=\exp \left[i\left(\alpha_{x}^{\top} \hat{x}+\alpha_{p}^{\top} \hat{p}\right)\right], \quad \underline{\alpha} \in \mathbb{R}^{2 n} .
$$

In order to define the quantum Lyapunov exponent, we consider derivations on the algebra $\mathcal{W}$. We denote by $\delta_{\underline{\alpha}}$ the derivation defined as the generator 
of the automorphism $A \mapsto W(t \underline{\alpha}) A W(-t \underline{\alpha})$ for all $A \in \mathcal{W}$. Therefore we have

$$
\delta_{\underline{\alpha}}(A) \equiv\left[L_{\underline{\alpha}}, A\right], \quad \forall A \in \mathcal{W},
$$

where $[$,$] is the commutator and$

$$
L_{\underline{\alpha}}=\alpha_{x}^{\top} \hat{x}+\alpha_{p}^{\top} \hat{p}, \quad \underline{\alpha} \in \mathbb{R}^{2 n} .
$$

In particular, we can check that

$$
\left[L_{\underline{\alpha}}, W\left(\underline{\alpha}^{\prime}\right)\right]=-\sigma\left(\underline{\alpha}, \underline{\alpha}^{\prime}\right) W\left(\underline{\alpha}^{\prime}\right), \quad \forall \underline{\alpha}, \underline{\alpha}^{\prime} \in V .
$$

We assume that the dynamics defines an automorphism of $\mathcal{W}$ :

$$
U^{\dagger}\left(t, t_{0}\right) A U\left(t, t_{0}\right) \equiv A\left(t, t_{0}\right) \in \mathcal{W}, \quad \forall A \in \mathcal{W}, \forall t, t_{0} \in \mathbb{R},
$$

where $U\left(t, t_{0}\right)$ denotes the unitary propagator with initial time $t_{0}$.

Definition 1 (cf. [6])

The upper quantum Lyapunov exponent is defined as

$$
\bar{\lambda}=\sup _{\underline{\alpha} \in V} \bar{\lambda}_{\underline{\alpha}}
$$

where

$$
\bar{\lambda}_{\underline{\alpha}}\left(U, L_{\underline{\alpha}}, A, t_{0}\right):=\limsup _{t \rightarrow \infty} \frac{1}{t} \ln \left\|\left[L_{\underline{\alpha}}, A\left(t, t_{0}\right)\right]\right\|,
$$

and $\|$.$\| is the norm of the C^{*}$-algebra $\mathcal{W}$.

Since the time evolution is unitary, the exponent $\bar{\lambda}_{\underline{\alpha}}$ can also be expressed as

$$
\bar{\lambda}_{\underline{\alpha}}\left(U, L_{\underline{\alpha}}, A, t_{0}\right)=\limsup _{t \rightarrow \infty} \frac{1}{t} \ln \left\|\left[L_{\underline{\alpha}}\left(t_{0}, t\right), A\right]\right\|,
$$

with

$$
L_{\underline{\alpha}}\left(t_{0}, t\right):=U^{\dagger}\left(t_{0}, t\right) L_{\underline{\alpha}} U\left(t_{0}, t\right) \text {. }
$$

\section{Definition 2}

A system satisfies the quantum Anosov relations [1, 24, 20], if there are $2 n$ directions $\underline{\alpha}_{1}, \ldots, \underline{\alpha}_{2 n} \in V$ such that the corresponding derivations satisfy for all $t, t_{0} \in \mathbb{R}$

$$
U\left(t, t_{0}\right) L_{\underline{\alpha}_{i}} U^{\dagger}\left(t, t_{0}\right)=e^{\lambda_{i}\left(t-t_{0}\right)} L_{\underline{\alpha}_{i}}
$$

where $\lambda_{i}$ are $2 n$ complex numbers such that

$$
\operatorname{Re}\left(\lambda_{1}\right) \leq \ldots \leq \operatorname{Re}\left(\lambda_{n}\right)<0<\operatorname{Re}\left(\lambda_{n+1}\right) \leq \ldots \leq \operatorname{Re}\left(\lambda_{2 n}\right) .
$$




\section{Remark 1}

We have extend the definition of [1] by allowing the numbers $\lambda_{i}$ to have an imaginary part. Moreover, we do not require that a state invariant under the actions of $H$ and $L_{\underline{\alpha}_{i}}$ exist.

\section{Remark 2}

To describe particles with internal structure, such as spin, it is necessary to generalize this construction. Assuming that the internal states of the particle form a complex Hilbert space $\mathfrak{h}$, the $C^{*}$-algebra $\mathcal{W}$ of canonical commutation relations over $\mathfrak{h}$ is by definition the $C^{*}$-algebra generated by elements $\{W(\underline{\alpha}) \mid \underline{\alpha} \in \mathfrak{h}\}$ such that

$$
\begin{aligned}
W(\underline{\alpha})^{\dagger} & =W(-\underline{\alpha}), \\
\left.W(\underline{\alpha}) W(\underline{\alpha})^{\prime}\right) & =e^{-\frac{i}{2} \operatorname{Im}\left(\left\langle\underline{\alpha}, \underline{\alpha}^{\prime}\right\rangle\right)} W\left(\underline{\alpha}+\underline{\alpha}^{\prime}\right),
\end{aligned}
$$

where $\langle.,$.$\rangle denotes the scalar product in \mathfrak{h}$.

\section{Quantum skew-product systems and enlarged Hilbert space}

A quantum skew-product system is described by the following Schrödinger equation with a non autonomous Hamiltonian in a Hilbert space $\mathcal{H}$ :

$$
i \frac{d}{d t} \phi(t)=H\left(\varphi^{t}(\theta)\right) \phi(t),
$$

where $\varphi^{t}$ is a continuous flow on a compact metric space $\mathcal{M}$ while $H(\theta)$ is a self-adjoint operator depending on the parameter $\theta \in \mathcal{M}$ such that the evolution operator $U\left(t, t_{0} ; \theta\right)$ exists and is strongly continuous with respect to $\theta \in \mathcal{M}$. This form of Hamiltonian operator includes periodic, quasiperiodic and almost-periodic time dependence according to whether $\mathcal{M}$ is a circle, a torus or the hull of an almost-periodic function.

Any solution of (3) can be written as

$$
\phi(t ; \theta)=U\left(t, t_{0} ; \theta\right) \phi\left(t_{0} ; \theta\right),
$$

with the operator $U\left(t, t_{0} ; \theta\right)$ satisfying

$$
i \frac{\partial}{\partial t} U\left(t, t_{0} ; \theta\right)=H\left(\varphi^{t}(\theta)\right) U\left(t, t_{0} ; \theta\right)
$$

and $U\left(t_{0}, t_{0} ; \theta\right)=\mathbb{1}_{\mathcal{H}}$.

The uniqueness of solutions of (3) allows us to deduce the relations

$$
\begin{aligned}
U\left(t, t_{1} ; \theta\right) U\left(t_{1}, t_{0} ; \theta\right) & =U\left(t, t_{0} ; \theta\right), \\
U\left(t+\tau, t_{0}+\tau ; \theta\right) & =U\left(t, t_{0} ; \varphi^{\tau}(\theta)\right),
\end{aligned}
$$


for all $t, t_{0}, t_{1}, \tau \in \mathbb{R}$ and all $\theta \in \mathcal{M}$.

Let $\mu$ be an invariant probability measure on $\mathcal{M}$. The family of Koopman operators $\left(\mathcal{T}^{t}\right)_{t \in \mathbb{R}}$, defined by

$$
\left(\mathcal{T}^{t} \psi\right)(\theta)=\psi\left(\varphi^{t}(\theta)\right) \quad \text { for all } \quad \psi \in \mathbb{L}^{2}(\mathcal{M}, d \mu)
$$

is a strongly continuous one-parameter unitary group of operators. According to Stone's theorem, there exists a self-adjoint operator $G$ which is an infinitesimal generator of $\mathcal{T}^{t}$ :

$$
\mathcal{T}^{t}=e^{i t G} \quad \text { for all } t \in \mathbb{R} .
$$

The separable Hilbert space $\mathcal{K}=\mathbb{L}^{2}(\mathcal{M}, d \mu ; \mathcal{H})=\mathbb{L}^{2}(\mathcal{M}, d \mu) \otimes \mathcal{H}$ will be called the enlarged space of $\mathcal{H}$. The family of operators $U\left(t, t_{0} ; \theta\right) \in \mathcal{H}$ depending on the parameter $\theta \in \mathcal{M}$ defines a unitary operator acting in $\mathcal{K}$ which maps a function $\theta \mapsto \psi(\theta) \in \mathcal{H}$ of $\mathcal{K}$ to the function $\theta \mapsto$ $U\left(t, t_{0} ; \theta\right) \psi(\theta) \in \mathcal{H}$. To avoid a complicated notation, we also denote this operator by $U\left(t, t_{0} ; \theta\right)$. Moreover, we omit the identity factor of $\mathcal{T}^{t} \otimes \mathbb{1}_{\mathcal{H}}$ in the Koopman operator in $\mathcal{K}$. From the uniqueness of solutions of (3) we can conclude that

$$
\mathcal{T}^{s} U\left(t, t_{0} ; \theta\right)=U\left(t, t_{0} ; \varphi^{s}(\theta)\right) \mathcal{T}^{s}
$$

for all $t, t_{0}, s \in \mathbb{R}$ and all $\theta \in \mathcal{M}$.

\section{Definition 3}

We define a unitary operator $U_{K}\left(t, t_{0}\right)$ acting on the enlarged space $\mathcal{K}$ by

$$
U_{K}\left(t, t_{0}\right)=\mathcal{T}^{-t} U\left(t, t_{0} ; \theta\right) \mathcal{T}^{t_{0}}=\mathcal{T}^{-\left(t-t_{0}\right)} U\left(t-t_{0}, 0 ; \theta\right) .
$$

One can show that it is strongly continuous in $t-t_{0}$, and Stone's theorem implies that there is a self-adjoint operator $K$ on $\mathcal{K}$, called generalized Floquet Hamiltonian, such that

$$
U_{K}\left(t, t_{0}\right)=e^{-i\left(t-t_{0}\right) K} .
$$

The solution of the associated Schrödinger equation

$$
i \frac{d}{d t} \psi(t)=K \psi(t)
$$

reads $\psi(t)=U_{K}\left(t, t_{0}\right) \psi\left(t_{0}\right) \in \mathcal{K}$, and it is linked to a solution $\phi$ of the Schrödinger equation (3) in $\mathcal{H}$ by

$$
\phi(t)=\mathcal{T}^{t} \psi(t)=\psi\left(t, \varphi^{t}(\theta)\right) .
$$




\section{Proposition 1}

We denote $H(\theta)$ the self-adjoint operator on $\mathcal{K}$ which maps $\psi \in \mathcal{K}$ to the function $\theta \mapsto H(\theta) \psi(\theta) \in \mathcal{H}$ of $\mathcal{K}$. We assume that $H(\theta)$ is a self-adjoint operator of $\mathcal{K}$. We have the formal equality

$$
K=G+H(\theta) .
$$

Proof:

The operator $U_{K}\left(t, t_{0}\right)$ is strongly differentiable on $\mathcal{D}(K)$, and we can write formally

$$
i \frac{\partial}{\partial t} U_{K}\left(t, t_{0}\right)=K U_{K}\left(t, t_{0}\right) \quad \text { for all } t, t_{0} \in \mathbb{R} \text {. }
$$

Therefore

$$
\begin{aligned}
K & =\left.i \frac{\partial}{\partial t} U_{K}\left(t, t_{0}\right)\right|_{t=t_{0}} \\
& =\left.i \frac{\partial}{\partial t}\left(\mathcal{T}^{-\left(t-t_{0}\right)} U\left(t-t_{0}, 0 ; \theta\right)\right)\right|_{t=t_{0}} \\
& =\left.i \frac{\partial}{\partial t}\left(\mathcal{T}^{-\left(t-t_{0}\right)}\right)\right|_{t=t_{0}} U(0,0 ; \theta)+\left.i \frac{\partial}{\partial t} U\left(t, t_{0} ; \theta\right)\right|_{t=t_{0}} \\
& =G+H(\theta) .
\end{aligned}
$$

\section{Quantum skew-product Anosov properties}

For a quantum skew-product system defined by the Schrödinger equation (3) with an Hamiltonian of the form $H\left(\hat{x}, \hat{p}, \varphi^{t}(\theta)\right)$, we define the Anosov property by

\section{Definition 4}

A quantum skew-product system satisfies the quantum skew-product Anosov relations if there exist $2 n$ functions $\underline{\alpha}_{1}, \ldots, \underline{\alpha}_{2 n}: \mathcal{M} \rightarrow V$ such that the corresponding derivations satisfy for all $t, t_{0} \in \mathbb{R}$ and $\theta \in \mathcal{M}$

$$
U\left(t, t_{0} ; \theta\right) L_{\underline{\alpha}_{i}\left(\varphi^{t} 0(\theta)\right)} U^{\dagger}\left(t, t_{0} ; \theta\right)=e^{\lambda_{i}\left(t-t_{0}\right)} L_{\underline{\alpha}_{i}\left(\varphi^{t}(\theta)\right)},
$$

where $\lambda_{i}$ are $2 n$ complex numbers such that

$$
\operatorname{Re}\left(\lambda_{1}\right) \leq \ldots \leq \operatorname{Re}\left(\lambda_{n}\right)<0<\operatorname{Re}\left(\lambda_{n+1}\right) \leq \ldots \leq \operatorname{Re}\left(\lambda_{2 n}\right) .
$$

The operators $L_{\underline{\alpha}_{i}(\theta)}$ define operators $L_{\underline{\alpha}_{i}}$ acting on the enlarged Hilbert space $\mathcal{K}=L^{2}(\mathcal{M}, \mu) \otimes \mathcal{H}$, given by

$$
\left(L_{\underline{\alpha}_{i}} \psi\right)(\theta)=L_{\underline{\alpha}_{i}(\theta)} \psi(\theta) \quad \text { for all } \quad \psi \in \mathcal{D}\left(L_{\underline{\alpha}_{i}}\right) \subset \mathcal{K} \text {. }
$$




\section{Proposition 2}

In the enlarged space $\mathcal{K}=\mathbb{L}^{2}(\mathcal{M}, \mu) \otimes \mathcal{H}$, the dynamics generated by the Hamiltonian $K=G+H(\theta)$ satisfies the standard quantum Anosov properties [1]:

$$
U_{K}\left(t, t_{0}\right) L_{\underline{\alpha}_{i}} U_{K}^{\dagger}\left(t, t_{0}\right)=e^{\lambda_{i}\left(t-t_{0}\right)} L_{\underline{\alpha}_{i}} .
$$

Proof:

By definition, the evolution operator satisfies

$$
U_{K}\left(t, t_{0}\right)=\mathcal{T}^{-t} U\left(t, t_{0} ; \theta\right) \mathcal{T}^{t_{0}}
$$

Then the equation

$$
U_{K}\left(t, t_{0}\right) L_{\underline{\alpha}_{i}} U_{K}^{\dagger}\left(t, t_{0}\right)=e^{\lambda_{i}\left(t-t_{0}\right)} L_{\underline{\alpha}_{i}}
$$

can be written as

$$
\mathcal{T}^{-t} U\left(t, t_{0}\right) \mathcal{T}^{t_{0}} L_{\underline{\alpha}_{i}} \mathcal{T}^{-t_{0}} U\left(t, t_{0} ; \theta\right) \mathcal{T}^{t}=e^{\lambda_{i}\left(t-t_{0}\right)} L_{\underline{\alpha}_{i}} .
$$

Using the relation

$$
\mathcal{T}^{t} L_{\underline{\alpha}_{i}(\theta)}=\mathcal{T}^{t} \alpha_{i_{x}}(\theta)^{\top} \otimes \hat{x}+\mathcal{T}^{t} \alpha_{i_{p}}(\theta)^{\top} \otimes \hat{p}=L_{\underline{\alpha}_{i}\left(\varphi^{t}(\theta)\right)} \mathcal{T}^{t}
$$

for all $t \in \mathbb{R}$, we obtain

$$
U\left(t, t_{0} ; \theta\right) L_{\underline{\alpha}_{i}\left(\varphi^{t_{0}}(\theta)\right)} U^{\dagger}\left(t, t_{0} ; \theta\right)=e^{\lambda_{i}\left(t-t_{0}\right)} L_{\underline{\alpha}_{i}\left(\varphi^{t}(\theta)\right)} .
$$

\section{Examples}

\subsection{The almost-periodic quantum parametric oscillator}

As in [6], we consider the parametric quantum oscillator which is described by the Hamiltonian (we take the mass $=1$ ):

$$
H(t)=\frac{1}{2} \hat{p}^{2}+\frac{1}{2} f(t) \hat{x}^{2}
$$

where $f$ is an almost-periodic real valued function.

The classical dynamics corresponding to the Hamiltonian (5) has the same form as the eigenvalue equation of the almost-periodic Schrödinger operator:

$$
-\ddot{x}+V(t) x=E x
$$

with $f(t)=E-V(t)$. For a fixed almost-periodic real valued function $V(t)$, we will now analyze the one-parameter family of systems defined by varying $E$ on $\mathbb{C}$ and, in particular, when $E$ is real and in the resolvent set $\rho$ of the almost-periodic Schrödinger operator $-d^{2} / d t^{2}+V(t)$. 


\section{Theorem 1}

For any observable $A=W(\beta)$ in the Weyl algebra, in the instability region $E \in \rho \cap \mathbb{R}$, there is a stable direction $\underline{\alpha}_{s}$, which depends on $t_{0}$, for which

$$
\bar{\lambda}_{\underline{\alpha}_{s}}\left(U, L_{\underline{\alpha}_{s}}, A, t_{0}\right)=-\lambda_{c}<0,
$$

whereas for all other directions $\underline{\alpha}$

$$
\bar{\lambda}_{\underline{\alpha}}\left(U, L_{\underline{\alpha}}, A, t_{0}\right)=\lambda_{c}>0 .
$$

where $\lambda_{c}$ is the Lyapunov exponent of the classical system. Thus the upper quantum Lyapunov exponent is positive,

$$
\bar{\lambda}=\sup _{\underline{\alpha}} \bar{\lambda}_{\underline{\alpha}}=\lambda_{c}>0 .
$$

\section{Proof:}

The spectral parameter $E$ is in the resolvent set $\rho$ of the operator if and only if the classical system

$$
\frac{d}{d t}\left(\begin{array}{c}
p \\
q
\end{array}\right)=\left(\begin{array}{cc}
0 & V(t)-E \\
1 & 0
\end{array}\right)\left(\begin{array}{l}
p \\
q
\end{array}\right)
$$

has an exponential dichotomy [8]. In particular, if $E \in \rho$, the system (6) has two linearly independent solutions $q_{+} \in \mathbb{L}^{2}\left(\left[0,+\infty[)\right.\right.$ and $\left.\left.q_{-} \in \mathbb{L}^{2}(]-\infty, 0\right]\right)$. The functions

$$
m_{ \pm}=\frac{p_{ \pm}}{q_{ \pm}} \quad \text { and } \quad \tilde{m}_{ \pm}=\frac{p_{ \pm}}{q_{ \pm}+i p_{ \pm}}
$$

defined for $E \notin \mathbb{R}$ and $E \in \rho \cap \mathbb{R}$, respectively, are almost-periodic [23, 10].

The classical Lyapunov exponent associated with the dynamics of (6) is defined as

$$
\lambda_{c}=\sup \left(\limsup _{t \rightarrow+\infty} \frac{1}{2 t} \ln \left(|p|^{2}+|q|^{2}\right)\right),
$$

where the supremum is taken over all non trivial solutions $(p, q)$ of (17), and it satisfies [

$$
\begin{aligned}
\lambda_{c} & =-\limsup _{t \rightarrow+\infty} \frac{1}{2 t} \ln \left(\left|p_{+}\right|^{2}+\left|q_{+}\right|^{2}\right) \\
& =\limsup _{t \rightarrow+\infty} \frac{1}{2 t} \ln \left(\left|p_{-}\right|^{2}+\left|q_{-}\right|^{2}\right)
\end{aligned}
$$

In order to determine the upper quantum Lyapunov exponent, we first need to calculate $L_{\underline{\alpha}}\left(t_{0}, t\right)$ which we write in the form

$$
L_{\underline{\alpha}}\left(t, t_{0}\right)=\alpha_{x}\left(t, t_{0}\right) \hat{x}+\alpha_{p}\left(t, t_{0}\right) \hat{p} .
$$


The propagator $F\left(t, t_{0}\right)$ of the classical equation (7), defined by

$$
\left(\begin{array}{c}
p(t) \\
x(t)
\end{array}\right)=F\left(t, t_{0}\right)\left(\begin{array}{c}
p\left(t_{0}\right) \\
x\left(t_{0}\right)
\end{array}\right), \quad F(t, t)=1 \quad \forall t
$$

may be written as

$$
F\left(t, t_{0}\right)=P(t)\left(\begin{array}{cc}
\frac{\psi_{+}(t)}{\psi_{+}\left(t_{0}\right)} & 0 \\
0 & \frac{\psi_{-}(t)}{\psi_{-}\left(t_{0}\right)}
\end{array}\right) P\left(t_{0}\right)^{-1}
$$

where $\psi_{ \pm}(t)=q_{ \pm}(t)+i p_{ \pm}(t)$ and

$$
P(t)=\left(\begin{array}{cc}
\tilde{m}_{+}(t) & \tilde{m}_{-}(t) \\
1-i \tilde{m}_{+}(t) & 1-i \tilde{m}_{-}(t)
\end{array}\right)
$$

Using the fact that the Heisenberg equations of motion for the operators $\hat{x}(t)$ and $\hat{p}(t)$ have the same form as the classical equations for $x(t)$ and $p(t)$, we can write

$$
\left(\begin{array}{c}
U^{\dagger}\left(t, t_{0}\right) \hat{p} U\left(t, t_{0}\right) \\
U^{\dagger}\left(t, t_{0}\right) \hat{x} U\left(t, t_{0}\right)
\end{array}\right)=F\left(t, t_{0}\right)\left(\begin{array}{c}
\hat{p} \\
\hat{x}
\end{array}\right)
$$

Thus, using the relation

$$
L_{\underline{\alpha}}\left(t, t_{0}\right)=\left(\begin{array}{c}
\alpha_{p} \\
\alpha_{x}
\end{array}\right)^{\top}\left(\begin{array}{c}
U^{\dagger}\left(t, t_{0}\right) \hat{p} U\left(t, t_{0}\right) \\
U^{\dagger}\left(t, t_{0}\right) \hat{x} U\left(t, t_{0}\right)
\end{array}\right)=\left(\begin{array}{c}
\alpha_{p}\left(t, t_{0}\right) \\
\alpha_{x}\left(t, t_{0}\right)
\end{array}\right)^{\top}\left(\begin{array}{c}
\hat{p} \\
\hat{x}
\end{array}\right),
$$

we obtain

$$
\left(\begin{array}{c}
\alpha_{p}\left(t, t_{0}\right) \\
\alpha_{x}\left(t, t_{0}\right)
\end{array}\right)=\left(P\left(t_{0}\right)^{-1}\right)^{\top}\left(\begin{array}{cc}
\frac{\psi_{+}(t)}{\psi_{+}\left(t_{0}\right)} & 0 \\
0 & \frac{\psi_{-}(t)}{\psi_{-}\left(t_{0}\right)}
\end{array}\right) P(t)^{\top}\left(\begin{array}{c}
\alpha_{p} \\
\alpha_{x}
\end{array}\right) .
$$

If $A=W(\beta)=e^{i\left(\beta_{x} \hat{x}+\beta_{p} \hat{p}\right)}$ then, according to (11),

$$
\left[L_{\underline{\alpha}}\left(t_{0}, t\right), A\right]=\left(\alpha_{p}\left(t_{0}, t\right) \beta_{x}-\alpha_{x}\left(t_{0}, t\right) \beta_{p}\right) A=-\sigma\left(\underline{\alpha}\left(t_{0}, t\right), \underline{\beta}\right) A,
$$

implying that

$$
\left\|\left[L_{\underline{\alpha}}\left(t_{0}, t\right), A\right]\right\|=\left|\alpha_{p}\left(t_{0}, t\right) \beta_{x}-\alpha_{x}\left(t_{0}, t\right) \beta_{p}\right|=\left|\sigma\left(\underline{\alpha}\left(t_{0}, t\right), \underline{\beta}\right)\right|,
$$

where we have used $\|A\|=1$. By (11), the stable direction $\underline{\alpha}_{s}$ is given by

$$
\left(\begin{array}{c}
\alpha_{p s} \\
\alpha_{x s}
\end{array}\right)=\left(\begin{array}{c}
-q_{+}\left(t_{0}\right) \\
p_{+}\left(t_{0}\right)
\end{array}\right) \in \mathbb{R}^{2}
$$

Indeed we obtain

$$
\left(\begin{array}{c}
\alpha_{p s}\left(t_{0}, t\right) \\
\alpha_{x s}\left(t_{0}, t\right)
\end{array}\right)=\psi_{+}(t)\left(\begin{array}{c}
-1+i \tilde{m}_{+}(t) \\
\tilde{m}_{+}(t)
\end{array}\right),
$$


and

$$
\left\|\left[L_{\underline{\alpha}_{s}}\left(t_{0}, t\right), A\right]\right\|=\left|\left(1+i \tilde{m}_{+}(t)\right) \beta_{x}+\tilde{m}_{+}(t) \beta_{p}\right|\left|\psi_{+}(t)\right| .
$$

According to (8), the quantum Lyapunov exponent in this direction is

$$
\begin{aligned}
\lambda_{\underline{\alpha}_{s}}\left(U, L_{\underline{\alpha}_{s}}, A, t_{0}\right) & =\limsup _{t \rightarrow+\infty} \frac{1}{t} \ln \left(\left|\psi_{+}(t)\right|\right) \\
& =\limsup _{t \rightarrow+\infty} \frac{1}{2 t} \ln \left(\left|p_{+}(t)\right|^{2}+\left|q_{+}(t)\right|^{2}\right) \\
& =-\lambda_{c}<0 .
\end{aligned}
$$

For all other directions $\underline{\alpha} \in \mathbb{R}^{2}$, it is easy to check that the upper Lyapunov exponent is positive,

$$
\begin{aligned}
\lambda_{\underline{\alpha}}\left(U, L_{\underline{\alpha}}, A, t_{0}\right) & =-\limsup _{t \rightarrow+\infty} \frac{1}{t} \ln \left(\left|\psi_{+}(t)\right|\right) \\
& =-\limsup _{t \rightarrow+\infty} \frac{1}{2 t} \ln \left(\left|p_{+}(t)\right|^{2}+\left|q_{+}(t)\right|^{2}\right) \\
& =\lambda_{c}>0 .
\end{aligned}
$$

\section{Remark 3}

The result of Theorem 1 can be extend to the multidimensional case where

$$
H(t)=\frac{1}{2} \hat{p}^{2}+\frac{1}{2} \hat{x}^{\top} A(t) \hat{x}
$$

with $A(t)$ a real symmetric matrix depending almost-periodically on time. Writing $A(t)=E \mathbb{1}+V(t)$, the equations of motion of corresponding classical system have the same form as the eigenvalue equation of the Schrödinger operator $-d^{2} / d t^{2}+V(t)$. In the instability region $E \in \rho \cap \mathbb{R}$, there will be $n$ stable directions $\underline{\alpha}_{s}$, depending on $t_{0}$, with negative Lyapunov exponent, while they will de positive for the remaining directions. The main argument is again the exponential dichotomy in the resolvent set [9, 11].

To study of the Anosov properties for the almost-periodic quantum parametric oscillator, we formulate it as a quantum skew-product system,

$$
H\left(\varphi^{t}(\theta)\right)=\frac{1}{2} \hat{p}^{2}+\frac{1}{2} \tilde{f}\left(\varphi^{t}(\theta)\right) \hat{x}^{2}
$$

where $\tilde{f}$ is the extension of the almost-periodic function $f$ to a continuous function on its hull and $\varphi^{t}$ the associated minimal flow (see [10]). As before we introduce a parameter $E$ by writing $\tilde{f}\left(\varphi^{t}(\theta)\right)=E-V\left(\varphi^{t}(\theta)\right)$, and we 
denote the hull of the almost-periodic function by $\mathcal{M}$.

The corresponding classical system is now given by

$$
\frac{d}{d t}\left(\begin{array}{l}
p \\
x
\end{array}\right)=\left(\begin{array}{cc}
0 & E-V\left(\varphi^{t}(\theta)\right) \\
1 & 0
\end{array}\right)\left(\begin{array}{l}
p \\
x
\end{array}\right) .
$$

\section{Definition 5}

A linear system of differential equations

$$
y^{\prime}(t)=A\left(\varphi^{t}(\theta)\right) y(t)
$$

with $y(t) \in \mathbb{R}^{n}$ and $A(\theta)$ a matrix depending on $\theta \in \mathcal{M}$, is called reducible if it can be transformed into a system with constant coefficients

$$
z^{\prime}=C z
$$

by a transformation $y(t)=T\left(\varphi^{t}(\theta)\right) z(t)$ where $T(\theta)$ is a non singular matrix for all $\theta \in \mathcal{M}$.

\section{Remark 4}

The system (13) is reducible when the potential is quasi-periodic with frequencies satisfying a Diophantine condition ([12, 22, 19]).

\section{Theorem 2}

Let the classical system (13) be reducible. Then the corresponding quantum parametric oscillator satisfies the quantum skew-product Anosov properties for $E$ being in the resolvent set, $E \in \rho$ : there exist two measurable functions $\underline{\alpha}_{ \pm}: \mathcal{M} \rightarrow \mathbb{R}^{2}$ and $\lambda_{ \pm}$such that $\pm \operatorname{Re}\left(\lambda_{ \pm}\right)>0$ and

$$
U\left(t, t_{0} ; \theta\right) L_{\underline{\alpha}_{ \pm}\left(\varphi^{t}(\theta)\right)} U^{\dagger}\left(t, t_{0} ; \theta\right)=e^{\lambda_{ \pm}\left(t-t_{0}\right)} L_{\underline{\alpha}_{ \pm}\left(\varphi^{t}(\theta)\right)},
$$

with $L_{\underline{\alpha}_{ \pm}(\theta)}=\alpha_{x \pm}(\theta) \hat{x}+\alpha_{p \pm}(\theta) \hat{p}$.

\section{Proof:}

Using reducibility and the hyperbolic character of the flow of (13) in the resolvent set, we obtain

$$
F\left(t, t_{0} ; \theta\right)=g\left(\varphi^{t}(\theta)\right) \exp \left[\left(t-t_{0}\right)\left(\begin{array}{cc}
\lambda_{+} & 0 \\
0 & -\lambda_{+}
\end{array}\right)\right] g\left(\varphi^{t_{0}}(\theta)\right)^{-1}
$$

where $g$ is a is a non singular matrix for all $\theta \in \mathcal{M}$ and $\operatorname{Re}\left(\lambda_{+}\right) \geq 0$.

Consequently,

$$
\begin{aligned}
& \left(\begin{array}{c}
U^{\dagger}\left(t, t_{0} ; \theta\right) \hat{p} U\left(t, t_{0} ; \theta\right) \\
U^{\dagger}\left(t, t_{0} ; \theta\right) \hat{x} U\left(t, t_{0} ; \theta\right)
\end{array}\right)=F\left(t, t_{0} ; \theta\right)\left(\begin{array}{c}
\hat{p} \\
\hat{x}
\end{array}\right) \\
& \quad=g\left(\varphi^{t}(\theta)\right)\left(\begin{array}{cc}
e^{\left(t-t_{0}\right) \lambda_{+}} & 0 \\
0 & e^{-\left(t-t_{0}\right) \lambda_{+}}
\end{array}\right) g\left(\varphi^{t_{0}}(\theta)\right)^{-1}\left(\begin{array}{c}
\hat{p} \\
\hat{x}
\end{array}\right) .
\end{aligned}
$$


Swapping $t$ with $t_{0}$ in this equation and using the identity $U^{\dagger}\left(t, t_{0} ; \theta\right)=$ $U\left(t_{0}, t ; \theta\right)$, we obtain

$$
\begin{array}{r}
U\left(t, t_{0} ; \theta\right) L_{\underline{\alpha}\left(\varphi^{t_{0}}(\theta)\right)} U^{\dagger}\left(t, t_{0} ; \theta\right)=\left(\begin{array}{c}
\alpha_{p}\left(\varphi^{t_{0}}(\theta)\right) \\
\alpha_{x}\left(\varphi^{t_{0}}(\theta)\right)
\end{array}\right)^{\top}\left(\begin{array}{c}
U\left(t, t_{0} ; \theta\right) \hat{p} U^{\dagger}\left(t, t_{0} ; \theta\right) \\
U\left(t, t_{0} ; \theta\right) \hat{x} U^{\dagger}\left(t, t_{0} ; \theta\right)
\end{array}\right) \\
=\left(\begin{array}{c}
\alpha_{p}\left(\varphi^{t_{0}}(\theta)\right) \\
\alpha_{x}\left(\varphi^{t_{0}}(\theta)\right)
\end{array}\right)^{\top} g\left(\varphi^{t_{0}}(\theta)\right)\left(\begin{array}{cc}
e^{-\left(t-t_{0}\right) \lambda_{+}} & 0 \\
0 & e^{\left(t-t_{0}\right) \lambda_{+}}
\end{array}\right) g\left(\varphi^{t}(\theta)\right)^{-1}\left(\begin{array}{c}
\hat{p} \\
\hat{x}
\end{array}\right) .
\end{array}
$$

Thus, we can deduce the stable and unstable directions

$$
\left(\begin{array}{c}
\alpha_{p-}(\theta) \\
\alpha_{x-}(\theta)
\end{array}\right)=\left(g(\theta)^{-1}\right)^{\top}\left(\begin{array}{l}
1 \\
0
\end{array}\right) \quad \text { and } \quad\left(\begin{array}{c}
\alpha_{p+}(\theta) \\
\alpha_{x+}(\theta)
\end{array}\right)=\left(g(\theta)^{-1}\right)^{\top}\left(\begin{array}{l}
0 \\
1
\end{array}\right) .
$$

Writing $g=\left(g_{i j}\right)_{1 \leq i, j \leq 2}$, we obtain

$\left\{\begin{array}{l}\alpha_{p-}(\theta)=g_{22}(\theta) \operatorname{det}(g(\theta))^{-1} \\ \alpha_{x-}(\theta)=-g_{12}(\theta) \operatorname{det}(g(\theta))^{-1}\end{array}\right.$ and $\quad\left\{\begin{array}{l}\alpha_{p+}(\theta)=-g_{21}(\theta) \operatorname{det}(g(\theta))^{-1} \\ \alpha_{x+}(\theta)=g_{11}(\theta) \operatorname{det}(g(\theta))^{-1}\end{array}\right.$

with

$$
U\left(t, t_{0} ; \theta\right) L_{\underline{\alpha}_{ \pm}\left(\varphi^{t}(\theta)\right)} U^{\dagger}\left(t, t_{0} ; \theta\right)=e^{ \pm \lambda_{+}\left(t-t_{0}\right)} L_{\underline{\alpha}_{ \pm}\left(\varphi^{t}(\theta)\right)}
$$

\subsection{The configurational quantum cat system}

We consider a charged particle of mass $m=1$ constrained to move in a unit square with periodic boundary conditions (period 1) submitted to external periodic time dependent electromagnetic fields. It was shown in Ref. 25, 26, that the external fields can be chosen in such a way that the configuration space of the particle is mapped periodically to itself according to Arnold's cat map. This system is described by the Hamiltonian

$$
H(\hat{x}, \hat{p}, t)=\frac{1}{2} \hat{p}^{\top} \hat{p}+\frac{1}{2}\left(\hat{p}^{\top} A+A^{\top} \hat{p}\right)
$$

with $\hat{p}=\left(\begin{array}{c}\hat{p}_{1} \\ \hat{p}_{2}\end{array}\right)$ and $\hat{x}=\left(\begin{array}{c}\hat{x}_{1} \\ \hat{x}_{2}\end{array}\right)$.

The vector potential $A$ of the fields has the form

$$
A=V \hat{x} \Delta_{T, \varepsilon},
$$

where $\Delta_{T, \varepsilon}$ is a sequence of smooth kicks of period $T$ and duration $\varepsilon<<T$, while $V$ is a matrix such that $\exp (V)$ is Arnold's cat map:

$$
e^{V}=C=\left(\begin{array}{ll}
2 & 1 \\
1 & 1
\end{array}\right) \text {. }
$$


The time evolution operator over one period $T$ or Floquet operator $U_{F}=$ $U(T, 0)$ becomes in the limit $\varepsilon \rightarrow 0$ :

$$
U_{F}=e^{-\frac{i T}{2} \hat{p}^{2}} e^{-\frac{i}{2}\left(\hat{x}^{\top} V^{\top} \hat{p}+\hat{p}^{\top} V \hat{x}\right)} .
$$

Since the configuration space of the system is a torus (as opposed to $\mathbb{R}^{n}$ in the former examples), we need to slightly adapt the definitions of Section 2. We choose as algebra $\mathcal{A}$ of observables the $C^{*}$-algebra generated by the Weyl operators $W(\beta, \gamma)=\exp \left[i\left(\beta^{\top} \hat{x}+\gamma^{\top} \hat{p}\right)\right]$ with $\beta \in 2 \pi \mathbb{Z}^{2}$ and $\gamma \in \mathbb{R}^{2}$.

\section{Remark 5}

In the definition 2 of the Anosov property, we can use derivations that are not necessarily inner derivations, i.e. we do not need to impose $\underline{\alpha}_{x} \in 2 \pi \mathbb{Z}^{2}$. Indeed, according to (1) $\delta_{\underline{\alpha}}(A)=\left[L_{\underline{\alpha}}, A\right] \in \mathcal{A}$ for all $\underline{\alpha}=\left(\underline{\alpha}_{x}, \underline{\alpha}_{p}\right) \in \mathbb{R}^{2 n}$.

\section{Theorem 3}

The configurational quantum cat system satisfies quantum Anosov properties: There exist two stable directions $\underline{\alpha}_{1}$ and $\underline{\alpha}_{2}$,

$$
U_{F} L_{\underline{\alpha}_{i}} U_{F}^{\dagger}=e^{-\lambda} L_{\underline{\alpha}_{i}} \quad i=1,2,
$$

and two unstable directions $\underline{\alpha}_{3}$ and $\underline{\alpha}_{4}$,

$$
U_{F} L_{\underline{\alpha}_{i}} U_{F}^{\dagger}=e^{\lambda} L_{\underline{\alpha}_{i}} \quad i=3,4,
$$

where $\lambda>0$ is such that $e^{ \pm \lambda}$ are the eigenvalues of Arnold's cat map $C$.

\section{Proof:}

The operator $D_{V}=e^{-\frac{i}{2}\left(\hat{x}^{\top} V^{\top} \hat{p}+\hat{p}^{\top} V \hat{x}\right)}$, one of the factors of the evolution operator $U_{F}$, is a dilatation:

$$
\begin{aligned}
& D_{V}^{\dagger} \hat{x} D_{V}=e^{V} \hat{x}=C \hat{x} \\
& D_{V}^{\dagger} \hat{p} D_{V}=e^{-V^{\top}} \hat{p}=C^{-1} \hat{p} .
\end{aligned}
$$

The evolution of the position and momentum operators over one period $T$ is thus given by

$$
\begin{aligned}
& U_{F} \hat{x} U_{F}^{\dagger}=C^{-1} \hat{x}+T C^{-1} \hat{p} \\
& U_{F} \hat{p} U_{F}^{\dagger}=C \hat{p} .
\end{aligned}
$$

Equation (15) allows one to conclude that

$$
U_{F} L_{\left(0, \alpha_{p}\right)} U_{F}^{\dagger}=\alpha_{p}^{\top} C \hat{p},
$$

with $L_{\underline{\alpha}}=L_{\left(\alpha_{x}, \alpha_{p}\right)}=\alpha_{x}^{\top} \hat{x}+\alpha_{p}^{\top} \hat{p}$. Therefore $\underline{\alpha}_{1}=\left(0, v_{-}\right)$and $\underline{\alpha}_{3}=\left(0, v_{+}\right)$ are respectively stable and unstable directions, where $v_{ \pm}$are the eigenvectors of $C$ with $C v_{ \pm}=e^{ \pm \lambda} v_{ \pm}$. 
Using equations (14) and (15), we observe that

$$
\begin{aligned}
U_{F}\left[\left(C^{2}-I d\right) \hat{x}+T \hat{p}\right] U_{F}^{\dagger} & =\left(C^{2}-I d\right) C^{-1}(\hat{x}-T \hat{p})+T C \hat{p} \\
& =C^{-1}\left[\left(C^{2}-I d\right) \hat{x}+T \hat{p}\right] .
\end{aligned}
$$

Hence $\underline{\alpha}_{2}=\left(\left(C^{2}-I d\right) v_{+}, T v_{+}\right)$and $\underline{\alpha}_{4}=\left(\left(C^{2}-I d\right) v_{-}, T v_{-}\right)$are the second pair of stable and unstable directions.

\section{Remark 6}

The derivations $\delta_{\underline{\alpha}_{1}}$ and $\delta_{\underline{\alpha}_{3}}$ are inner derivations, but $\delta_{\underline{\alpha}_{2}}$ and $\delta_{\underline{\alpha}_{4}}$ are not because the coefficients of each eigenvector $v_{ \pm}$are rationally independent.

\section{Remark 7}

It follows immediately from the Anosov properties that the upper Lyapunov exponent for this system is $\bar{\lambda}=\lambda>0$.

\section{References}

[1] G. G. Emch, H. Narnhofer, W. Thirring, and G. L. Sewell. Anosov actions on noncommutative algebras. Journal of Mathematical Physics, 35(11):5582-5599, 1994.

[2] S. Guérin and H. R. Jauslin. Control of quantum dynamics by laser pulses: Adiabatic Floquet theory. Advances in Chemical Physics, 125:147-267, 2003.

[3] J. S. Howland. Two problems with time-dependent Hamiltonians. In Mathematical methods and applications of scattering theory, J.A. DeSanto, A. W. Saenz and W. W. Zachary, eds. Springer lecture Notes in Physics, v. 130, Springer-Verlag, New York, 1980, pp. 163-168.

[4] J. S. Howland. Stationary scattering theory for time-dependent Hamiltonians. Mathematische Annalen, 207:315-335, 1974.

[5] H. R. Jauslin and J. L. Lebowitz. Spectral and stability aspects of quantum chaos. Chaos, 1(1):114-121, 1991.

[6] H. R. Jauslin, O. Sapin, S. Guérin, and W. F. Wreszinski. Upper quantum Lyapunov exponent and parametric oscillators. Journal of Mathematical Physics, 45(11):4377-4385, 2004. 
[7] R. Johnson. Lyapunov numbers for the almost periodic Schrödinger equation. Illinois Journal of Mathematics, 28(3):54-78, 1984.

[8] R. Johnson. Exponential dichotomy, rotation number and linear differential operators with bounded coefficients. Journal of Differential Equations, 61:54-78, 1986.

[9] R. Johnson. $m$-functions and Floquet exponents for linear differential systems. Annali Matematica Pura ed Applicata, 147(4):211-248, 1987.

[10] R. Johnson and J. Moser. The rotation number for almost periodic potentials. Communications in Mathematical Physics, 84:403-438, 1982.

[11] R. Johnson and M. Nerurkar. Exponential dichotomy and rotation number for linear Hamiltonian systems. Journal of Differential Equations, 108(1):201-216, 1994.

[12] R. Johnson and G. R.Sell. Smoothness of spectral subbundles and reducibility of quasi-periodic linear differential system. Journal of Differential Equations, 41:262-288, 1981.

[13] W.A. Majewski. Does quantum chaos exist? A quantum Lyapunov exponents approach. e-print archive, 1998.

[14] W.A. Majewski and M. Kuna. On quantum characteristic exponents. Journal of Mathematical Physics, 34(11):5007-5015, 1993.

[15] V. I. Man'ko and R. Vilela Mendes. Lyapunov exponent in quantum mechanics. A phase-space approach. Physica D. Nonlinear Phenomena, 145:330-348, 2000.

[16] V. I. Man'ko and R. Vilela Mendes. Quantum sensitive dependence. Physics Letters A, 300:353-360, 2002.

[17] R. Vilela Mendes. Sensitive dependence in quantum systems: some examples and results. Physics Letters A, 171:253-258, 1992.

[18] R. Vilela Mendes. On the existence of quantum characteristic exponents. Physics Letters A, 187:299-301, 1994.

[19] J. Moser and J. Pöschel. An extension of a result by Dinaburg and Sinai on quasiperiodic potentials. Commentarii Mathematici Helvetici, 59:39-85, 1984.

[20] H. Narnhofer. Kolmogorov systems and Anosov systems in quantum theory. Infinite Dimensional Analysis, Quantum Probability and Related Topics, 4(1):85-119, 2001. 
[21] I.J. Peter and G. G. Emch. Quantum Anosov flows: A new family of examples. Journal of Mathematical Physics, 39(9):4513-4539, 1998.

[22] R. Sacker and G. Sell. A spectral theory for linear differential systems. Journal of Differential Equations, 27(2):320-358, 1978.

[23] G. Scharf. Fastperiodische Potentiale. Helvetica Physica Acta, 24:573605, 1965.

[24] W. Thirring. What are the quantum mechanical Lyapunov exponents? In H. Grosse et. al., editor, Low-dimensional models in statistical physics and quantum field theory. Prooceedings of the 34 Internationale Universitätswoche für Kern- und Teilchenphysik, Schladming, Austria, 4-11 March, 1995, pages 223-237. Lecture Notes in Physics, 469, Springer, Berlin, 1996.

[25] S. Weigert. The configurational quantum cat map. Zeitschrift für Physik B. Condensed Matter, 80(1):3-4, 1990.

[26] S. Weigert. Quantum chaos in the configurational quantum cat map. Physical Review A. Third Series, 48(3):1780-1798, 1993.

[27] S. Weigert. Quantum parametric resonance. Journal of Physics A. Mathematical and General, 35(18):4169-4181, 2002.

[28] K. Yajima. Scattering theory for Schrödinger equations with potentials periodic in time. Journal of the Mathematical Society of Japan, 29:729 $743,1977$.

[29] K. Yajima. Resonances for the AC-Stark effect. Communications in Mathematical Physics, 87:331-352, 1982.

[30] K. Yajima. Bound states and scattering states for time periodic Hamiltonians. Annales de l'institut Henri Poincaré. Section A. Physique Théorique, 39(2):145-157, 1983. 